\title{
Transport and drag in undoped electron-hole bilayers
}

\author{
E. H. Hwang and S. Das Sarma \\ Condensed Matter Theory Center, Department of Physics, \\ University of Maryland, College Park, Maryland 20742-4111
}

(Dated: November 1, 2018)

\begin{abstract}
We investigate transport and Coulomb drag properties of semiconductor-based electron-hole bilayer systems. Our calculations are motivated by recent experiments in undoped electron-hole bilayer structures based on GaAs-AlGaAs gated double quantum well systems. Our results indicate that the background charged impurity scattering is the most dominant resistive scattering mechanism in the high-mobility bilyers. We also find that the drag transresistivity is significantly enhanced when the electron-hole layer separation is small due to the exchange induced renormalization of the single layer compressibility.

PACS numbers: 73.40.-c, 73.21.Ac, 71.30.+h
\end{abstract}

\section{INTRODUCTION}

Much interest has recently focused on bilayer semiconductor structures $\frac{1,2,3}{2}$, where two quantum wells are put in close proximity with a high insulating barrier between them to suppress interlayer tunneling. Such systems are of intrinsic interest because competition between intralayer and interlayer Coulomb interaction (i.e., correlation effects), particularly at low carrier densities when Coulomb interaction dominates (or in high magnetic fields, when the electronic kinetic energy is quenched), may lead to exotic ground states, collective properties, and quantum phase transitions. Although much of the recent work has concentrated on bilayer quantum Hall systems ${ }^{2}$, the essential issue of the competition between electron kinetic energy and intra-/interlayer Coulomb correlations is quite generic, and interactioninduced novel ground states and collective properties are possible even in the zero field situation if the carrier density is low enough and the two layers are close enough to produce strong inter-layer interaction.

Significant recent progress ${ }^{3}$ has been made in the fabrication of two dimensional (2D) electron-hole bilayers because of intense interest in exciton condensation effects that are predicted to occur at low carrier densities and close proximity of the two-dimensional electron gas (2DEG) to the two-dimensional hole gas $(2 \mathrm{DHG})^{4}$. At sufficiently low densities the attractive interlayer Coulomb interaction between an electron in one layer with a hole in the other layer would dominate over the intralayer repulsive interaction. An excitonic phase with electron-hole pairs (EHP) may form under such conditions, being lower in energy than the independent electron and hole Fermi liquid layers. Unlike excitons in bulk semiconductors, these EHPs would not annihilate by emitting radiation because the electrons and holes are spatially separated in two different layers.

Exciton condensation has previously been reported in electron or hole, but not electron-hole, bilayer quantum Hall systems ${ }^{2}$, but at a zero magnetic field the measurement of exciton effects in electron-hole (e-h) bilayers has proven to be extremely difficult because of the limitation of adjusting the densities in the two layers, in the low density regime, to match up the density of the 2DEG to the density of the $2 \mathrm{DHG}^{3}$. However, recent fabrication of e-h bilayer systems ${ }^{5.6}$ based on the heterostructure insulated-gate field-effect transistors (HIGFETs) $\underline{7.8}$ allows for independent contacts to each layer as well as high mobility and independently tunable low densities for the $2 \mathrm{DEG}$ and $2 \mathrm{DHG}$. Recent experiments ${ }^{6}$ in very low density and high quality e-h bilayers on HIGFETs show anomalous drag resistance behavior. The e-h bilayers on HIGFETs are undoped gated double quantum well devices, so the transport properties of the device may be different from the previously investigated modulation doped e-h bilayer systems ${ }^{3}$. Recent experiments ${ }^{5}$ have reported on the density dependent 2D mobilities of the electrons and holes in e-h bilayer in the same low density regime where the anomalous drag resistivity has been observed ${ }^{6}$.

Our goal in this paper is to develop detailed and realistic quantitative theories for density-dependent transport and drag in 2D e-h bilayers using the Fermi liquid picture for both carrier systems. For transport calculations, we consider Coulomb scattering by unintentional random charged impurities invariably present in the background. For drag calculations, we consider the Coulomb interaction between the electron and the hole layers. The important ingredient of physics included in both theoretical calculations is wave vector and frequency dependent dielectric screening by the electrons and holes themselves - for the charged impurity scattering induced transport calculation, the relevant screening is static since the impurities are quenched in the background whereas for the e-h frictional drag, the full dynamical screening must be considered in the theory. Our aim (and hope) in this work is to assist in the experimental detection of the excitonic phase in e-h bilayers through a careful comparison between our theory and the recent measurements 5.6 where any qualitative (or even dramatic quantitative) difference between our theory and the experimental data could provide suggestive evidence (or at least, interesting clues) for the failure of the Fermi liquid picture in the physical system, thereby indicating the possible emergence of a novel 
non-Fermi liquid collective phase.

In this paper, motivated by recent experiments on low-density e-h bilayers ${ }^{5.6}$, we study the transport properties of undoped electron-hole bilayers fabricated on HIGFETs. We calculate both the mobilities of each layer and the frictional Coulomb drag resistivity. In order to understand the low temperature $2 \mathrm{D}$ transport properties we have carried out a microscopic transport calculation using the Boltzmann theory ${ }^{9,10}$. Since the HIGFET is an undoped heterostructure, the 2D carrier resistivity is limited by screened background random Coulomb scattering in high mobility systems. With the unintentional background random 3D charged impurities as the main scattering center we obtain good agreement with the recent transport experiments ${ }^{-5}$ in undoped e-h bilayer systems.

In order to calculate the frictional drag resistivity of undoped electron-hole bilayers we use the many bodyFermi liquid diagrammatic perturbation theory with dynamically screened electron-electron interaction 1.11 . The recent experiments in electron-hole bilayers with small layer separations and especially at low temperatures and densities 6 show that the measured Coulomb drag increases as the temperature decreases at the lowest measured temperatures, which can not be explained based on the Fermi liquid theory, since the Fermi liquid theory predicts the low-temperature $T^{2}$ (or $T^{2} \ln T$ ) behavior of drag resistivity $\underline{-1}$. This anomalous behavior in the e-h drag may be related to the exciton condensation. However, our calculation shows good agreement with the experimentally observed drag data for the electron-hole bilayer with large separation, and even bilayers with small separation above the critical temperature of possible exciton condensation. The reasonable agreement between our theory and the measured $\operatorname{drag}^{\underline{6}}$ in the larger layer separation regime for all temperatures and the smaller separation regime for higher temperatures is in some sense tantalizing circumstantial evidence in support of the emergence of the collective excitonic phase in the measurements reported in ref. 6, where the drag shows a minimum as a function of temperature and increases with decreasing temperature at the lowest temperature.

We describe our background charged impurity limited transport theory and results in Sec. II, followed by our e-h Coulomb drag results in Sec. III. We conclude in Sec. IV with a discussion.

\section{TRANSPORT}

We start by writing down the theoretical formulae for conductivity $\sigma$ in the many-body Fermi liquid RPABoltzmann theory approximation widely used in the literature $\frac{9.10}{}$. The carrier conductivity $\sigma$ is given by $\sigma=n e \mu$, where $n$ is the carrier density and the carrier mobility $\mu$ is given by $\mu=e\langle\tau\rangle / m$. Here $m$ is the carrier effective mass and $\langle\tau(E)\rangle$ is the energy averaged transport relaxation time. We calculate the impurity ensemble averaged relaxation time $\tau(E)$ due to elastic disorder scattering by the background quenched charged impurities in the Born approximation:

$$
\begin{aligned}
\frac{1}{\tau\left(E_{k}\right)} & =\frac{2 \pi}{\hbar} \sum_{\alpha} \int \frac{d^{2} k^{\prime}}{(2 \pi)^{2}} \int_{-\infty}^{\infty} d z N_{i}^{(\alpha)}(z) \\
& \times\left|u^{(\alpha)}\left(\mathbf{k}-\mathbf{k}^{\prime} ; z\right)\right|^{2}\left(1-\cos \theta_{\mathbf{k k}^{\prime}}\right) \delta\left(E_{k}-E_{k^{\prime}}\right)(1)
\end{aligned}
$$

where $E(k)=\hbar^{2} k^{2} / 2 m$ is the $2 \mathrm{D}$ carrier energy for $2 \mathrm{D}$ wave vector $\mathbf{k} ; \theta_{\mathbf{k k}^{\prime}}$ is the scattering angle between carrier scattering wave vectors $\mathbf{k}$ and $\mathbf{k}^{\prime}$; the delta function $\delta\left(E_{k}-E_{k^{\prime}}\right)$ assures energy conservation for elastic scattering due to charged impurities where the screened scattering potential is denoted by $u^{(\alpha)}(\mathbf{q} ; z)$ with $\mathbf{q} \equiv \mathbf{k}-\mathbf{k}^{\prime}$ as the $2 \mathrm{D}$ scattering wave vector and $z$ is the quantization or the confinement direction normal to the $2 \mathrm{D}$ layer. The quantity $N_{i}^{(\alpha)}(z)$ in Eq. (11) denotes the 3D charged impurity density (with the $z$ dependence reflecting a possible impurity distribution) of the $\alpha$-th kind with $\alpha$ representing the various possible types of impurities which may be present in 2D semiconductor structures. However, we emphasize that there is no intentional doping in the HIGFETs used in refs. 5,6. Therefore, we use the unintentional random background 3D impurities as the only scattering source in our calculation to keep the number of unknown parameters a minimum - this background random 3D impurity density essentially sets the overall scale of resistivity in our results. We emphasize that we can obtain good qualitative agreement with experimental data by choosing three different kinds of charged impurities (i.e. interface, remote, and bulk) parameterized by a few reasonable parameters, but we do not see much point in this data fitting-type endeavor, and therefore keep only one unknown parameter in the theory, $n_{3 D}$, which is the $3 \mathrm{D}$ density of the unintentional charged impurities, which are assumed to be uniformly and randomly distributed the background throughout the whole sample. As emphasized above, this only lets the scale of the overall resistivity, not its qualitative behavior.

In Eq. (1) the screened impurity potential $u^{(\alpha)}(q ; z)$ is given by $u^{(\alpha)}(q ; z) \equiv V_{\mathrm{imp}}^{(\alpha)}(q ; z) / \epsilon(q)$ where $V_{\mathrm{imp}}^{(\alpha)}$ is the bare potential due to a charged impurity and $\epsilon(q)$ is the carrier dielectric screening function which is necessary since the charged impurity potential is Coulombic. The bare impurity potential is given by

$$
V_{\mathrm{imp}}^{(\alpha)}(q ; z)=\frac{2 \pi Z^{(\alpha)} e^{2}}{\kappa q} F_{\mathrm{imp}}^{(\alpha)}(q ; z),
$$

where $Z^{(\alpha)}$ is the impurity charge strength, $\kappa$ is the background (static) lattice dielectric constant, and $F_{\mathrm{imp}}$ is a form factor determined by the location of the impurity and the subband wavefunction $\psi(z)$ defining the $2 \mathrm{D}$ quantum well confinement. The finite wave vector dielectric screening function is written in the RPA as

$$
\epsilon(q)=1-v(q) \Pi(q, T),
$$

where $\Pi(q, T)$ is the $2 \mathrm{D}$ irreducible finite-temperature (and finite wave vector) polarizability function and 


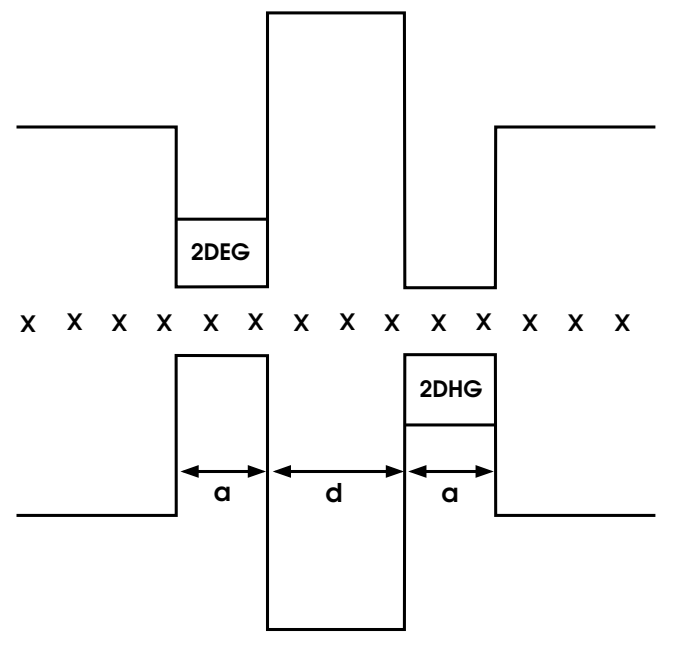

FIG. 1: The sample configuration used in this calculation is shown schematically. In this calculation we use the parameters $a=200 \AA, d=300 \AA$. "x" denotes the charged impurities which are distributed uniformly in the background with a $3 \mathrm{D}$ effective density of $n_{3 D}$.

$v(q)=v_{2 D}(q) f(q)$ is the effective bare electron-electron (Coulomb) interaction in the system with $v_{2 D}(q)=$ $2 \pi e^{2} /(\kappa q)$ being the 2D Fourier transform of the Coulomb potential, and $f(q)$ being the Coulomb form factor arising from the subband wavefunctions $\psi(z)$.

In Fig. 1 the sample configuration, following the experimental references ${ }^{5,6}$, used in our calculation is shown schematically. The electron and hole quantum wells with widths $a=200 \AA$ are separated by the $d=300 \AA$ barrier. Since there is no intentional doping, the charged Coulomb impurities are distributed randomly with a constant 3D impurity density $n_{3 D}$ throughout the sample (both barriers and quantum wells). Our sample configuration resembles the experimental setup of refs. 5,6 .

In Fig. 2 we show (a) the calculated density dependent mobility of each layer and (b) the ratio of hole mobility to electron mobility at fixed temperature $T=300$ $\mathrm{mK}$ for the sample configuration given in Fig. 1. The symbols indicate some representative experimental data points taken from ref. 5. We use a fixed charged impurity density $n_{3 D}=4.2 \times 10^{14} \mathrm{~cm}^{-3}$ and the known effective mass $m=0.067 m_{e}$ for electrons and $m=0.3 m_{e}$ for holes, respectively. As emphasized above the only resistive carrier scattering included in the results of Fig. 2 is that by background charged impurities. (We discuss later the issue of inter-layer e-h scattering itself contributing to $2 \mathrm{D}$ transport, arguing it to be negligibly small). We assume a single value of $n_{3 D}$ for calculating electron and hole mobilities in the different layers. We include the effects of confinement potential through the infinite square well confinement model, which should be excellent for the samples of refs. 5 and 6 . We neglect all phonon scattering effects because our theoretical estimate shows phonon scattering to be negligible for $2 \mathrm{D}$ carriers in GaAs structures in the $T<1 K$ regime of

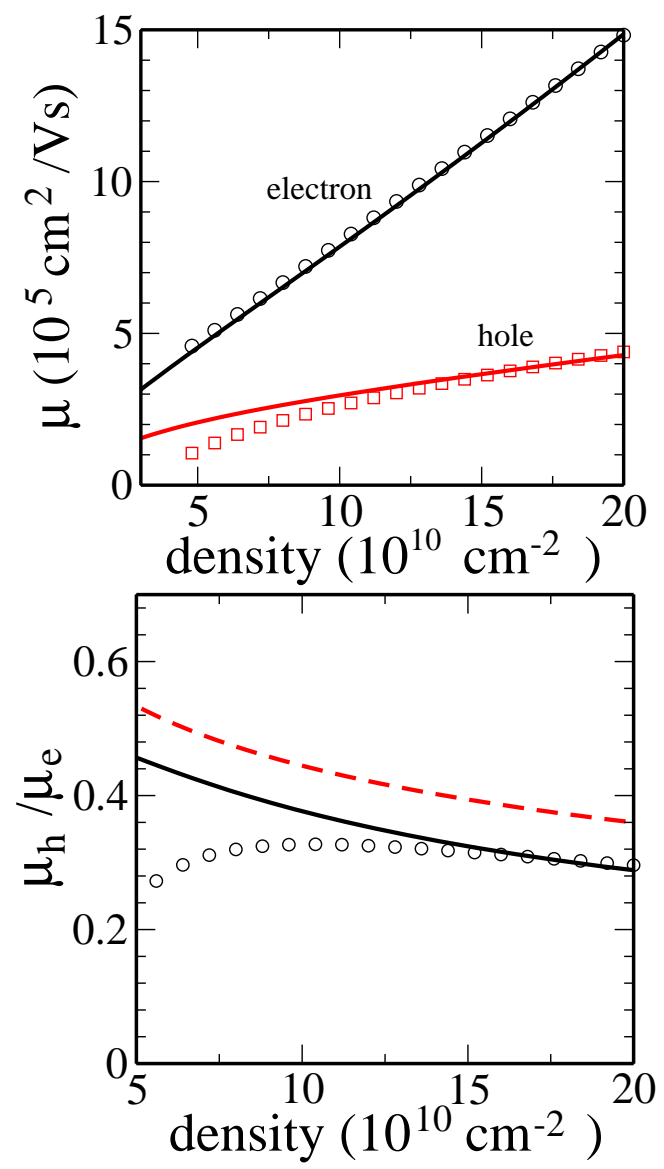

FIG. 2: (Color online) (a) Calculated mobilities of electron $(m=0.067)$ and hole $(m=0.3)$ as a function of carrier density. The symbols are experimental data taken from ref. [5]. (b) The ratio of hole mobility to electron mobility. The solid lines are full theory and the dashed line in (b) is the simple approximation given in the Eq. (4) of the text. Here we use $n_{3 D}=4.2 \times 10^{14} \mathrm{~cm}^{-3}$.

interest to us. The phonon scattering becomes relevant typically in the $T>3 K$ regime $\frac{12}{2}$.

For the experimental density range $(n \sim 5 \times$ $10^{10} \mathrm{~cm}^{-2}-2 \times 10^{11} \mathrm{~cm}^{-2}$ ) our theoretically calculated density dependent electron mobility has an effective exponent $\alpha \approx 1$ in $\mu \propto n^{\alpha}$, which is consistent with the experimental data of ref. 5, indicating that the screened background charged impurity is the main scattering source in the sample. For the density dependent hole mobility we can not explain the experimental data for all densities by a single power law behavior. At high hole densities, $p>10^{11} \mathrm{~cm}^{-2}$ the calculated mobility approaches an approximate power law behavior $\alpha \approx 0.7$, which is in excellent agreement with the experimental data of ref. 5. At low hole densities, $p<10^{11} \mathrm{~cm}^{-2}$, the power law changes to a much stronger function of density, pointing to a change in the screening properties of the system at low carrier density. Although our theoretical result in Fig. 2(a) indicates a changing exponent $\alpha$ for the holes at lower $\left(<10^{11} \mathrm{~cm}^{-2}\right)$ hole densities, the 
experimental $\alpha$ is much more strongly affected by the decreasing density than our RPA Boltzmann transport theory. We emphasize that the discrepancy between the low density experimental data and our calculation indicates that the screening theory, which captures the essential experimental features at higher densities, does not describe the hole transport very well at the lower range of the experimental density.

There are several understandable reasons for the systematic discrepancy between our theory and the lower density hole data. First, the holes, being more massive $\left(m_{h}=0.3 m\right.$ versus $\left.m_{e}=0.07 m\right)$, have stronger interaction effects than electrons as characterized by the dimensionless interaction coupling strength $r_{s}=(\pi n)^{-1 / 2} / a_{B}$ where $a_{B}=\kappa \hbar^{2} / m e^{2}$ is the effective Bohr radius, which for the holes varies between $r_{s} \approx 12-6\left(n_{h}=5 \times 10^{10}-\right.$ $2 \times 10^{11} \mathrm{~cm}^{-2}$ ) compared with $r_{s} \approx 2.7-1.3$ for the electrons. The large value of the hole interaction parameter $r_{s} \sim 12$ at the hole density of $5 \times 10^{10} \mathrm{~cm}^{-2}$ indicates that our RPA-Boltzmann theory may be less quantitatively reliable at lower hole densities than at lower electron densities since the theory is really quantitatively valid only at small $r_{s}$, where, of course, we get excellent agreement with the experimental data both for electrons and holes. Second, and perhaps more importantly, charged impurities introduce strong density inhomogeneity at low carrier densities as linear screening eventually breaks down leading to a $2 \mathrm{D}$ metal to $2 \mathrm{D}$ insulator transition (2D MIT $)^{13,14}$, thereby causing a systematic failure of our linear screening RPA-Boltzmann theory at low enough densities. While such a density inhomogeneity driven failure of our linear RPA-Boltzmann theory is inevitable at low enough carrier densities for both the electrons and the holes, the critical density for the hole $2 \mathrm{D} \mathrm{MIT}^{14}$ is typically much larger than the corresponding electron critical density 13 . We thus expect that our RPA-Boltzmann theory will deviate more from the experimental data for 2D holes than for 2D electrons at lower carrier densities, particularly since the $2 \mathrm{D}$ holes have lower mobilities than the $2 \mathrm{D}$ electrons. We believe that this nonlinear screening effect along with the large $r_{s}$ values are responsible for the discrepancy between theory and experiment for the low density hole results shown in Fig. 2.

In Fig. 2(b) we show the ratio of hole mobility to electron mobility as a function of carrier density. In the high density regime, $n>10^{11} \mathrm{~cm}^{-2}$, the measured experimental mobility ratio is about 0.3 and decreases gradually as density increases. Our calculated $\mu_{h} / \mu_{e}$ shows excellent agreement with experimental data in the high density limit, but deviates from the experiment at low $\left(<10^{11} \mathrm{~cm}^{-2}\right)$ density for reasons discussed above (i.e. large $r_{s}$ and density inhomogeneity). For screened Coulomb scattering, assuming strong screening, we can easily derive the approximate qualitative formula for the mobility by ignoring the wave vector dependence in Eq. (1) for transport:

$$
\mu \propto\left(2 k_{F}+q_{T F}\right)^{2} / m^{2},
$$

where $k_{F}=(2 \pi n)^{1 / 2}$ is the Fermi wave vector and $q_{T F}=$ $2 / a_{B}$ is the Thomas Fermi screening wave vector. Thus, in the high density limit $\left(q_{T F} / 2 k_{F} \ll 1\right.$, typically $n>$ $10^{13} \mathrm{~cm}^{-2}$ for GaAs systems) the mobility is proportional to the inverse square of the effective mass, (i.e. $\mu \propto$ $1 / m^{2}$ ). However, in the low density limit (or $q_{T F} / 2 k_{F} \gg$ 1 ) the mobility is independent of the carrier mass. Thus, in the simple theory the ratio of mobility increases to unity as density decreases. But the measured mobility ratio decreases as density decreases below $n<10^{11} \mathrm{~cm}^{-2}$. Again this experimental behavior arises from nonlinear screening and the large $r_{s}$ values at low carrier density.

In Fig. 2(b), in addition to the full numerically calculated RPA-Boltzmann transport results and the experimental results from ref. 5, we also show the simple results for $\mu_{h} / \mu_{e}$ derived on the basis of the qualitative analytic formula of Eq. (4). It is quite interesting to note that in the experimental density range covered in the measurements of ref. 5, the measured $\mu_{h} / \mu_{e} \approx 0.28-0.32$ is almost a density independent constant, which does not differ much from the simple electron-hole effective mass ratio $\mu_{h} / \mu_{e} \approx m_{e} / m_{h} \approx 0.23$ which is very different from either the high density $\left(q_{T F} \ll 2 k_{F}\right)$ asymptotic value $\mu_{h} / \mu_{e}=\left(m_{e} / m_{h}\right)^{2} \approx 0.05$ or the low density $\left(q_{T F} \gg 2 k_{F}\right)$ asymptotic value $\mu_{h} / \mu_{e} \approx 1$. The disagreement of the approximate formula, Eq. (4), with the experimental data can be easily understood by the realizing that the dimensionless parameter $q_{T F} / 2 k_{F}=r_{s} / \sqrt{2}$ changes between 0.92 and 1.9 for electrons and 4.2 and 8.5 for holes in the experimental density range of Fig. 2, and therefore neither the strong screening nor the weak screening approximation applies. It is, however, curious that the actual experimental (and the full theoretical) values of $\mu_{h} / \mu_{e} \sim m_{e} / m_{h}$ in the experimental density range studied in Fig. 2, which (i.e. $\mu \propto \sigma \propto m^{-1}$ ) would follow trivially from the Drude conductivity formula $\sigma=n e^{2} \tau / m$ if one assumes $\tau$ to be a constant independent of effective mass as is often done in the literature. This apparent approximate agreement of the trivial $\mu_{h} / \mu_{e} \sim m_{e} / m_{h}$ relationship with the experimental results of ref. 5 is, however, purely fortuitous, and should not be taken seriously. For very high (low) electron and hole density, there is no doubt that $\mu_{h} / \mu_{e} \sim m_{e}^{2} / m_{h}^{2}$ $(\sim 1)$, but both the asymptotic high and low density regimes would be difficult to achieve experimentally, and in smoothly going between these two regimes, the intermediate density regime of experimental interest only seems to obey the simple $\mu_{h} / \mu_{e} \sim m_{e} / m_{h}$ relation since this $m_{e} / m_{h}$ behavior is obviously intermediate between $\left(m_{e} / m_{h}\right)^{2}$ and $\left(m_{e} / m_{h}\right)^{0}=1$.

It is, however, interesting to note that the approximate mobility formula defined by Eq. (4), where the charged impurity scattering strength is characterized by the constant momentum transfer of $\left(2 k_{F}+q_{T F}\right)$ completely ignoring the wave vector dependence of Coulomb interaction and assuming Thomas-Fermi screening so that the $1 / q$ Coulomb scattering strength is parameterized simply by $1 /\left(2 k_{F}+q_{T F}\right)$ corresponding to the $q=2 k_{F}$ backward 
carrier scattering across the 2D Fermi surface, describes well the density dependence of mobility as compared with the full RPA-Boltzmann theory. Both results disagree with experiments at low carrier densities for reasons discussed above.

\section{COULOMB DRAG}

The principal motivation ${ }^{5,6}$ behind trying to fabricate closely spaced e-h bilayers with small interlayer separation is to probe the interlayer e-h Coulomb interaction. The bilayer frictional drag, which is a direct probe of interlayer correlations, in a many body-Fermi liquid diagrammatic perturbation theory with dynamically screened electron-electron interaction is given by 1,3

$$
\rho_{D}=\frac{\hbar^{2}}{2 \pi e^{2} n p k_{B} T} \int \frac{q^{2} d^{2} q}{(2 \pi)^{2}} \int d \omega \frac{F_{e}(q, \omega) F_{h}(q, \omega)}{\sinh ^{2}(\beta \omega / 2)},
$$

where $F_{e, h}(q, \omega)=\left|u_{e h}^{s c}(q, \omega)\right| \operatorname{Im} \prod_{\mathrm{e}, \mathrm{h}}(\mathrm{q}, \omega)$, with $u_{e h}^{s c}=$ $v_{e h}^{c} / \epsilon(q, \omega)$ is the dynamically screened interlayer Coulomb interaction between the electron and the hole layers, and $\Pi(q, \omega)$ is the $2 \mathrm{D}$ polarizability. Note that the dielectric function $\epsilon(q, \omega)$ entering Eq. (5) is a 2component tensor and is given by 15

$$
\begin{aligned}
|\epsilon(q, \omega)| & =\left[1-v_{e}(q) \Pi_{e}(q, \omega)\right]\left[1-v_{h}(q) \Pi_{h}(q, \omega)\right] \\
& -v_{e h}(q) v_{h e}(q) \Pi_{e}(q, \omega) \Pi_{h}(q, \omega),
\end{aligned}
$$

where $e, h$ correspond to electron and hole layers. (For details on the drag formula and its implications, see refs. 1.3.) With the assumption of a large inter-layer separation $d\left(k_{F} d \gg 1, q_{T F} d \gg 1\right)$ it is easy to show that within RPA, where vertex corrections are neglected in the dynamical polarizability $\Pi$, the drag resistivity is given at low temperatures $\left(T \ll T_{F}\right)$ by the simple formula:

$$
\rho_{D}=\frac{m_{e} m_{h}}{n p e^{2}} \frac{\zeta(3)}{16 \hbar} \frac{\left(k_{B} T\right)^{2}}{k_{F_{e}} k_{F_{h}} q_{T F_{e}} q_{T F_{h}} d^{4}}
$$

with $\zeta$ being the Riemann zeta function. This result shows that $\rho_{D}(T, d) \propto T^{2} / d^{4}$. However, we note that Eq. (7) is valid only for high density and low temperature $\left(T / T_{F} \ll 1\right)$ as well as large separation. At low densities or small layer separations (i.e. $k_{F} d \lesssim 1$ ) the actual drag is much enhanced compared with the simple formula of Eq. (7) as has earlier been discussed 11 .

In our calculation we include the finite layer thickness effect and relax the condition $k_{F} d \gg 1$ because $k_{F}$ and $d$ are small in the samples of ref. 6 - this makes large momentum scattering important in contrast to the usual $k_{F} d \gg 1$ case where carrier backscattering is neglected. In Coulomb drag between two high density 2D electron layers with large layer separation (i.e. $k_{F} d \gg 1$ ) the backward scattering $q \approx 2 k_{F}$ is ignored due to the exponential dependence of the interlayer Coulomb interaction $V(q) \propto \exp (-q d) / q$ on $d$. In this case the Coulomb drag is dominated by small angle scattering and Eq. (7) is
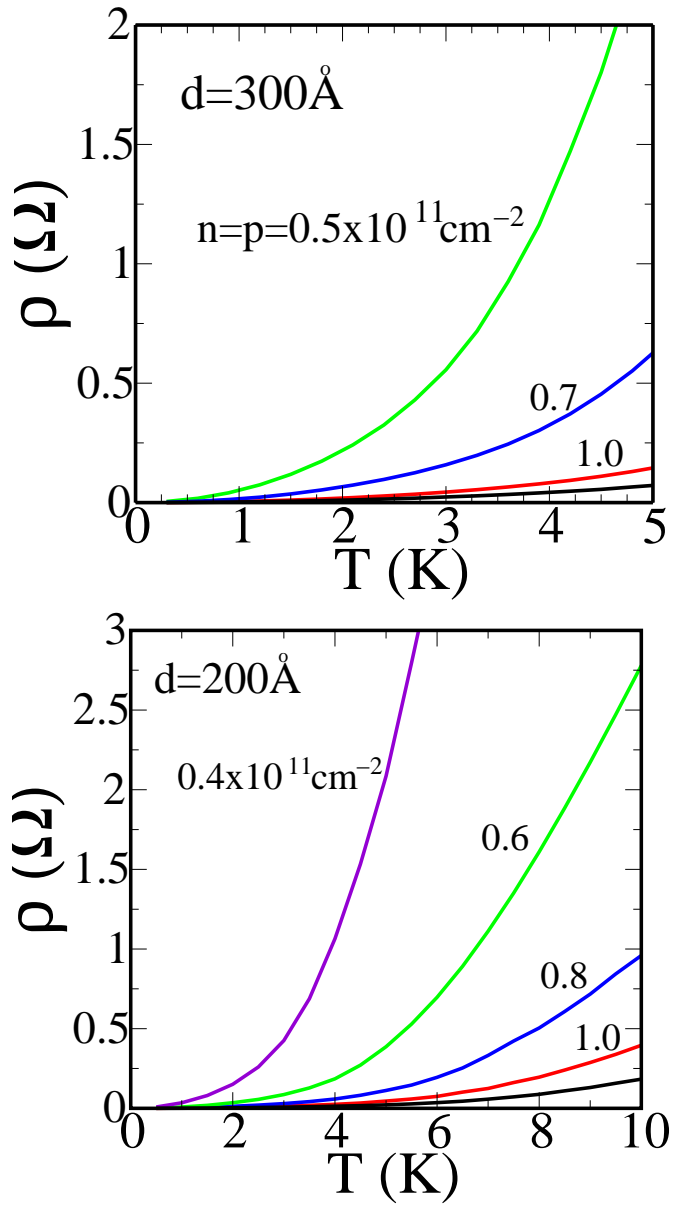

FIG. 3: (Color online) Calculated Coulomb drag of electronhole bilayer systems with (a) $d=300 \AA$ barrier and (b) $d=200 \AA$ barrier. In (a) we use the matched electronhole density of $n=p=5,7,10,12 \times 10^{10} \mathrm{~cm}^{-2}$ and in (b) $n=p=4,6,8,10,12 \times 10^{10} \mathrm{~cm}^{-2}$.

a very good approximation. In the e-h bilayer of small $d$ (and low density) in ref. 6, large angle scattering becomes important and Eq. (7) would not apply at all, and one needs to carry out the full calculation of Eqs. (5) and (6) to obtain the e-h drag.

We directly theoretically calculate the e-h Coulomb drag using Eqs. (5) and (6) without making any additional assumptions and using the sample parameters of refs. 5 and 6 . In Fig. 3 we show the calculated drag resistivity of electron-hole bilayer with each well width of $a=200 \AA$ as a function of temperature for several matched e-h densities. Here the electron layer is the driving layer and the hole layer is the drag layer, i.e. the current $I_{e}$ is applied in the electron layer and drag voltage $V_{D}$ measured in the hole layer with $\rho_{D}=V_{D} / I_{e}$. In Fig. 3(a) we use the density $n=p=5,7,10,12$ $\times 10^{10} \mathrm{~cm}^{-2}$ and a layer separation of $d=300 \AA$. In the experiments of ref. 6 the observed low density drag resistivity $\left(n=p=5 \times 10^{10} \mathrm{~cm}^{-2}\right)$ is orders of magnitude larger than the corresponding theoretical drag result of 
Eq. (7), but our calculation with Eq. (5) shows good agreement with the experimental data within a factor of 2. In e-h bilayers the low-energy acoustic plasmon lies inside the single particle excitation region of the heavier carriers ${ }^{16}$, which gives rise to a tremendous enhancement of drag resistivity not captured at all in Eq. (7). We include this effect by going beyond the simple RPA (valid at high density) in calculating the polarizability by incorporating vertex corrections through local field corrections. The strong correlation effect in the low densities gives to a large enhancement of the drag due to the presence of coupled plasmon modes.

In Fig. 3(b) we use the density $n=p=4,6,8,10$, $12 \times 10^{10} \mathrm{~cm}^{-2}$ and a layer separation of $d=200 \AA$. With this narrow barrier sample, Seamons et $a l \underline{\underline{6}}$ find very interesting and unexpected drag resistivity which is not observed in the $d=300 \AA$ barrier sample of ref. 6 . Even though the measured drag resistivity show $\rho_{D} \propto T^{2}$ behavior above $T \approx 1 K$, for temperatures below $1 \mathrm{~K}$ an upturn of the Coulomb drag is measured with decreasing temperatures (instead of $\rho_{D} \rightarrow 0$ as $T^{2}, \rho_{D}$ increases as $T \rightarrow 0$ ). The characteristic temperature below which the observed $\rho_{D}$ increases anomalously in ref. 6 with decreasing temperature itself increases with density. Thus, the upturn in drag at low temperature can not be explained within our Fermi liquid theory and may signal the formation of a novel phase in electron-hole system. However our calculation at temperatures above $T=1 K$ shows good agreement with the experimental data and we also get good agreement at all temperatures for sample with larger $d$, neither of which would happen with the simple formula of Eq. (7) which disagrees with experiments by orders of magnitude.

\section{DISCUSSION AND CONCLUSION}

Before conclusion, we return to transport and discuss the layer independence of transport in the electron-hole bilayer. This layer independence is reflected in the experimental observation ${ }^{5.6}$ of the mobility in one layer (e.g. the electron layer) to be independent of the carrier density in the other layer (i.e. the hole layer) and vice versa. In the presence of the adjacent layer the total scattering time of one layer can be expressed as $1 / \tau_{t}=1 / \tau_{i}+1 / \tau_{D}$, where $\tau_{i}$ is the scattering time due to the background charged impurities given in Eq. (1) and shown in Fig.2, and $\tau_{D}$ is the e-h scattering time due to the inter-carrier inter-layer scattering between electrons and holes which is given by $\tau_{D}=m / n e^{2} \rho_{D}$, with $\rho_{D}$ being our calculated inter-layer e-h frictional drag resistivity [Eq. (5) and Fig. 3]. However, for samples we consider in this paper the typical scattering times are $\tau_{i} \sim n s$ and $\tau_{D} \sim \mu s$ below $T=2 K$. i.e. $\tau_{i}^{-1} \gg \tau_{D}^{-1}$. Therefore we expect the mobility of each layer to be dominated by impurity scattering and entirely independent of the adjacent layer density. The extremely small measured and calculated values of the interlayer Coulomb drag $\rho_{D}$, corresponding to extremely long drag relaxation times of microseconds, imply that interlayer e-h scattering makes a very small $(\sim 0.1 \%)$ contribution to the measured $d c$ conductivity (i.e. 2D mobility) of each layer. The measured 2D mobility in the experimental e-h bilayers of refs. 5 and 6 is therefore entirely dominated by the background charged impurity scattering. As an immediate consequence of this finding $\left(\tau_{D} \gg \tau_{i}\right)$ we predict, that the measured $2 \mathrm{D}$ electron (hole) mobility in each layer would be completely independent of the carrier density in the other layer. This prediction has indeed been explicitly verified experimentally $\underline{\underline{6}}$.

In conclusion, we theoretically study transport and frictional drag of undoped electron-hole bilayers based on HIGFETS within a many-body Fermi liquid theory. We find that the unintentional background charged impurity scattering is the most dominant resistive scattering mechanism in the recent experimental systems ${ }^{5,6}$. This implies that the 2D mobility in each layer is independent of the carrier density in the other layer in the high-mobility structures of refs. 5,6. We also find that the drag resistivity is significantly enhanced when the electron-hole layer separation is small, but our Fermi liquid many-body approach cannot explain the recently observed $\underline{\underline{6}}$ upturn in the drag resistance with the lowering of temperature, which may be indicating the emergence of a non-Fermi liquid excitonic phase in closely spaced bilayers. Our Fermi liquid theory would always predict $\rho_{D} \rightarrow 0$ as $T \rightarrow 0$, and $\rho_{D}$ increasing with decreasing $T$ is an unexplained anomaly in the experimental observation of Ref. 6.

This work is supported by DOE through Sandia National Laboratories.
1 A. P. Jauho and H. Smith, Phys. Rev. B 47, 4420 (1993); L. Zheng and A. H. MacDonald, Phys. Rev. B 48, 8203 (1993); K. Flensberg and B. Y. K. Hu, Phys. Rev. B 52, 14796 (1995); T. J. Gramila, J. P. Eisenstein, A. H. MacDonald, L. N. Pfeiffer, and K. W. West, Phys. Rev. B 47, 12957 (1993)

2 J. P. Eisenstein and A. H. MacDonald, Nature (London) 432, 691 (2004).

3 U. Sivan, P. M. Solomon, and H. Shtrikman, Phys. Rev.
Lett. 68, 1196 (1992); B. E. Kane, J. P. Eisenstein, W. Wegscheider, L. N. Pfeiffer, and K. W. West, Appl. Phys. Lett. 65, 3266 (1994); J. A. Keogh, K. Das Gupta, H. E. Beere, D. A. Ritchie, and M. Pepper, Appl. Phys. Lett. 87, 202104 (2005).

4 S. I. Shevchenko, Sov. J. Low Temp. Phys. 2, 251 (1976).

5 J. A. Seamons, D. R. Tibbetts, J. L. Reno, and M. P. Lilly, Appl. Phys. Lett. 90, 052103 (2007); C. P. Morath, J. A. Seamons, J. L. Reno, and M. P. Lilly, arXiv:0803.1402 
6 J. A. Seamons, C. P. Morath, J. L. Reno, and M. P. Lilly, preprint (2008).

7 B. E. Kane, L. N. Pfeiffer, K. W. West, and C. K. Harnett, Appl. Phys. Lett. 63, 2132 (1993); B. E. Kane, L. N. Pfeiffer, and K. W. West, Appl. Phys. Lett. 67, 1262 (1995).

8 M. P. Lilly, J. L. Reno, J. A. Simmons, I. B. Spielman, J. P. Eisenstein, L. N. Pfeiffer, K. W. West, E. H. Hwang, and S. Das Sarma, Phys. Rev. Lett. 90, 056806 (2003).

9 S. Das Sarma and E. H. Hwang, Phys. Rev. Lett. 83, 164 (1999); Phys. Rev. B 61, R7838 (2000); 69, 195305 (2004).

10 T. Ando, A. B. Fowler, and F. Stern, Rev. Mod. Phys. 54, 437 (1982)

11 E. H. Hwang, S. Das Sarma, V. Braude, and A. Stern, Phys. Rev. Lett. 90, 086801 (2003); S. Das Sarma and E.
H. Hwang, Phys. Rev. B 71, 195322 (2005).

12 S. Das Sarma and E. H. Hwang, unpublished.

13 S. Das Sarma, M. P. Lilly, E. H. Hwang, L. N. Pfeiffer, K. W. West, and J. L. Reno, Phys. Rev. Lett. 94, 136401 (2005).

14 M. J. Manfra, E. H. Hwang, S. Das Sarma, L. N. Pfeiffer, K. W. West, and A. M. Sergent, Phys. Rev. Lett. 99, 236402 (2007); R. Leturcq, D. L'Hote, R. Tourbot, C. J. Mellor, and M. Henini, Phys. Rev. Lett. 90, 076402 (2003).

15 S. Das Sarma and E. H. Hwang, Phys. Rev. Lett. 84, 5596 (2000).

16 S. Das Sarma and A. Madhukar, Phys. Rev. B 23, 805 (1981); J. K. Jain and S. Das Sarma, Phys. Rev. B 36, 5949 (1987). 